\title{
The Past, Current, and Future Application of Cryopreserved Human Hepatocytes in Drug Development
}

\section{Xiao Mei Zhuang ${ }^{1}$ and Chuang $\mathrm{Lu}^{2 *}$}

${ }^{1}$ State Key Laboratory of Toxicology and Medical Countermeasures, Beijing Institute of Pharmacology and Toxicology, Beijing, China ${ }^{2}$ Department of Drug Metabolism and Disposition, Sanofi, Massachusetts, USA

\begin{abstract}
The liver is the major organ responsible for metabolizing xenobiotics, including many therapeutic drugs. A hepatocyte is the main cell type in the liver where metabolism occurs. As intact cells, the cell membrane of a hepatocyte contains various transporters to regulate the drug uptake and efflux and, therefore, the intracellular drug concentration and the rate of metabolism and potential of hepatoxicity. A drug could diffuse through the membrane passively or be up taken actively by transporters such as organic anion transporting peptide (OATP) and sodium taurocholate co-transporting polypeptide (NTCP) into the hepatocytes [1]. Once the drug enters the hepatocytes, the efflux pumps, such as the multidrug resistance protein transporters (MRPs or MDR1), may pump the drug out of the hepatocytes to reduce the intracellular concentration. Hepatocytes also carry all the drug metabolizing enzymes and cofactors at their physiological concentrations and provide a drug metabolism environment that closely mimics that of in vivo. Freshly isolated primary human hepatocytes are the gold standard for drug metabolism and transporter studies because they maintain most of the activities of hepatocytes packed in the liver. Due to the existence of species difference in drug transport and metabolism [2], human hepatocytes are usually the optimal choice in drug development for human therapeutic drugs.
\end{abstract}

However, there are some limitations to the application of freshly isolated hepatocytes in drug discovery experiments. For example, 1) they are not readily available; the tissues hepatocytes are harvested from are usually refused ones from liver transplants due to the need for freshness (time after procurement prior to isolation of hepatocytes) and the pathological conditions of the liver, such as high fat content, cirrhosis, etc., 2) Each preparation can be used only once because, by their nature, hepatocytes lose their viability in several hours when cultured in suspension, or in several days when cultured in a plated format on support plastic and matrix surface. Thus, it is difficult to repeat a study in the same laboratory or compare studies between laboratories using freshly isolated hepatocytes from the same donor. 3) The quality of the hepatocytes, such as the levels of enzyme activity, cannot be characterized before they are used in studies, as some preparation (isolation) could result in poor quality (low activity) hepatocytes, or some livers may have already lost most of the activity prior the hepatocyte isolation. 4) Some donors may be poor metabolizers of certain polymorphic enzymes, thus the hepatocytes can not represent the general population, and researchers may generate results they cannot explain without knowing the genetic background of the donor. Therefore, being able to cryopreserve human hepatocytes will allow for the repeat use of the same precharacterized hepatocyte lots in different studies, either to screen many compounds over a long period of time to select the drug candidate or to conduct a complex and multistep experiments over a period of time. Cryopreserved hepatocytes also allow researchers from different labs to repeat and confirm each other's study results. For this reason, the FDA made a special recommendation in their 2012 drug-drug interaction guidance for pharmaceutical companies to use cryopreserved human hepatocytes in their CYP induction studies so the agency can better judge the study results as the same lots of cryopreserved hepatocytes may have been used in multiple

\section{Publication History:}

Received: December 04, 2016

Accepted: December 15, 2016

Published: December 17, 2016

\section{Keywords:}

Human hepatocytes, Cryopreservation, 3D culture, Drug metabolism, Cytochrome P450, Transporters

submissions, and the FDA may have tested results of positive control compounds themselves [3].

The isolation of rodent hepatocytes for drug metabolism study was reported as early as the 1970's [4,5]. Most human hepatocytes isolations and studies were in small scale and dependent on surgical biopsy tissue from local hospitals [6-8]. Due to the limited availability of quality tissue, usually due to the freshness and the condition of the tissue (discussed above), whole liver perfusion with digestive enzyme for hepatocyte isolation was not common before the1990's. Another technical challenge of whole liver perfusion is controlling the digestion time at $37^{\circ} \mathrm{C}$, where the digestion enzyme works but one must also simultaneously make sure the hepatocytes in the liver are not under hypoxia condition, as the hepatocytes consume more oxygen at $37^{\circ} \mathrm{C}$. After hepatocytes are isolated, cryopreservation of hepatocytes and then maintenance of their characteristic properties is often a challenge. For example, many lots of cryopreserved hepatocytes cannot be plated on plastic supported matrix surfaces, therefore cannot be cultured and used for some studies such as CYP induction or bile duct excretion. In the late 90's, a group of scientists reported success in isolation and cryopreservation of human hepatocytes from whole liver perfusion $[9,10]$. The development and then commercialization of cryopreserved human hepatocytes was a big advancement for its use as an in vitro system. The pre-characterized cryopreserved hepatocytes can now serve as a "reagent" and allow researchers to conduct experiments at their desired schedule and test drug candidates using the same lot(s) of hepatocytes over a period of time. This also allows for the same study to be compared between different laboratories, or for collaboration between different laboratories using the same experimental conditions. In addition, the researchers can choose specific pre-characterized lots (donors) to meet their research needs, such as studies in special populations including smokers, alcohol users, or poor metabolizers (e.g. CYP2D6 or CYP2C19). Because cryopreserved human hepatocytes are shown to preserve enzymatic and transporter activities, the FDA accepted it as a valid experimental

"Corresponding Author: Dr. Chuang Lu, Department of Drug Metabolism and Disposition, Sanofi, 153 second Avenue, Waltham, MA 02451, USA, Tel: 978257-9401; E-mail: chuanglu7@gmail.com

Citation: Zhuang XM, Lu C (2016) The Past, Current, and Future Application of Cryopreserved Human Hepatocytes in Drug Development. Int J Clin Pharmacol Pharmacother 1: 120. doi: https://doi.org/10.15344/2016/2456-3501/120

Copyright: (c) 2016 Zhuang et al. This is an open-access article distributed under the terms of the Creative Commons Attribution License, which permits unrestricted use, distribution, and reproduction in any medium, provided the original author and source are credited. 
model for metabolism and drug-drug interaction studies to support IND and NDA submission as early as 2006 [11]. Sequentially, in the 2012 DDI guidance [3], the FDA suggests the use of cryopreserved human hepatocytes, not freshly isolated, in the CYP induction studies to have a better control in the quality of hepatocytes used in those studies.

A recent advancement in the application of cryopreserved human hepatocytes in drug discovery is the adoption of a new incubation method, hepatocytes-suspension-in-human-plasma (HSIHP). Like many in vitro test systems, hepatocyte incubations are usually performed in a protein-free medium to avoid the complication of drug protein binding. Then, protein bindings in both in vitro and in vivo (plasma) are incorporated into the in vitro-in vivo extrapolation (IVIVE) [12]. Because of the uncertainty in determining the protein binding precisely in both in vitro incubations and in human plasma, and possible errors of incorporate multiple free fractions $\left(f_{u}\right)$ into the IVIVE, it is often challenging to have a reliable IVIVE, especially for high protein binding compounds with $\mathrm{f}_{\mathrm{u}}<0.01$. Due to this concern, the FDA suggests the use of an $f_{u}$ of 0.01 for any compounds which have protein binding greater than $99.9 \%$, just to be conservative in free drug exposure assessment [3]. Some researchers have used cryopreserved hepatocytes (or liver microsomes) suspended in plasma as an incubation method and found it improved the in vitroin vivo correlations (IVIVC) in drug clearance and inhibition-based drug-drug interaction (DDI) prediction [12-21]. In this approach, incubations that mimic the in vivo condition were applied to generate the drug intrinsic clearance or inhibition information without the need to generate the protein binding data from both hepatocyte incubation in vitro and plasma sample in vivo. This assay condition also reported better identifies kinetic parameters $\left(\mathrm{k}_{\mathrm{I}}\right.$ and $\left.\mathrm{K}_{\text {inact }}\right)$ for some time-dependent inhibitors that resulted in more accurately predicts clinical results from both single and repeat dose clinical trials [21].

Liu and colleagues [22,23] have modified the sandwich culture condition by adding a thick layer of Matrigel ${ }^{\mathrm{TM}}$ on top of the hepatocytes to study biliary excretion of compounds. This application provides a major advantage in that one can identify species similarities to find a preclinical species that might predict human in vivo biliary clearance. A few examples are reported in literature that demonstrate a good prediction of human biliary excretion using a cryopreserved human hepatocyte culture $[24,25]$. In the case where human hepatocytes are not readily available, rat hepatocytes under a similar culture condition were used to demonstrate the utility of studying Triptolide induced hepatotoxicity and CYP-P-gp interplay with several specific or dual CYP and P-gp inhibitors and inducers which modulated the intracellular concentration of triptolide and thence hepatotoxicity [26].

The traditional 2-dimetinal hepatocyte culture serves well for large scale and relatively fast screens of metabolic stability, metabolite profiling, DDI potential, hepatotoxicity, and transporter studies in the pharmaceutical industry. However, it tends to dedifferentiate after about 7-10 days in culture and results in loss of hepatic phenotypes and characteristics, including the morphology and metabolic/transporter activities. Recent development of cryopreserved hepatocyte applications has been focused on 3-dimensional culturing systems that theoretically are in a configuration closer to that of in vivo. It has been reported that primary hepatocytes and induced pluripotent stem (iPS) cells derived hepatocytes were cultured on tissue chips as $3 \mathrm{D}$ organoids, both in a static or perfusable platform. The perfuse rate could be adjusted on the tissue chip setting for the potential to culture different type of cells, such as hepatocytes as well as heart or lung cells. The culture hepatocyte hepatocytes 3D spheroids showed a lifetime of at least 28 days under perfusion with culture medium [27]. Nguyen et al., [28] applied bioprint technology and constructed a 3D liver tissue culture in a 24 -well Transwell ${ }^{\mathrm{R}}$ plate using primary human hepatocytes, human hepatic stellate cells, and human umbilical vein endothelial cells. They have showed that this $3 \mathrm{D}$ culture has high liver characteristic activities compared to a $2 \mathrm{D}$ culture. Using this $3 \mathrm{D}$ culturing system, they further demonstrated the hepatotoxicity upon trovafloxacin treatment, a withdrawn drug which is believed to have an inflammatory component involved in its hepatotoxicity in clinic. Human hepatocyte spheroids culture represents another direction of $3 \mathrm{D}$ culture. It has shown that the culture maintains in vivo hepatocyte morphology, viability, and hepatocyte-specific functions in a duration greater than 5 weeks [29]. The spheroids culture provided a relatively simple way to culture human hepatocytes which doesn't require any mechanic devices and special setups besides preparation of the spheroids. It provides potential for a longer period study for drug induced hepatotoxicity under chronic exposure of test drugs. Isolation and culture of Upcyte human hepatocytes (UHH, the premature of primary hepatocytes) is another approach for long term human hepatocyte culturing. Uncytes can have a proliferative capacity and can be cultured for approximately 35 passages (or, more precisely, doublings). For each passage, it can be culture for up to 18 days and it has comparable CYP, UGT, and sulfotransferases activities, except some reports show generally low CYP1A2 and high CYP2B6 activity levels in Upcyte cultures compared to primary hepatocyte cultures [30].

However, 3D cultures remain the emerging technology and the current focus of long term primary human hepatocytes culturing techniques to create an in vitro system more mimic to in vivo. A successful 3D culture could provide a test system for longer term chronic study for metabolism, transporter activity, or hepatotoxicity. The current systems are all still at the "beta testing' stage and most are in academic settings. The limitations of the current system for large scale applications in industry for drug screen including 1) the labor intensive set up for the fine turning of the culturing system, often needing specially designed mechanical devices; 2) the small scale at which it is applicable for an isolated mechanistic study, limiting its application as a general tool for testing or screening of drug candidates; and 3) that some devices carry a very high price tag that inhibits adoption by the industry.

\section{Competing Interests}

The authors declare that they have no competing interests.

\section{References}

1. Shitara Y, Li AP, Kato Y, Lu C, Ito K, et al. (2003) Function of uptake transporters for taurocholate and estradiol 17beta-D-glucuronide in cryopreserved human hepatocytes. Drug Metab Pharmacokinet 18: 33-41.

2. Guengerich FP (2006) Cytochrome P450s and other enzymes in drug metabolism and toxicity. AAPS J 8: E101-111.

3. FDA (2012) Guidance for Industry Drug Interaction Studies-Study Design, Data Analysis, Implications for Dosing, and Labeling Recommendations.

4. Berry MN, Friend DS (1969) High-yield preparation of isolated rat liver parenchymal cells: a biochemical and fine structural study. J Cell Biol 43: 506-520.

5. Bojar H, Basler M, Fuchs F, Dreyfürst R, Staib W, et al. (1976) Preparation of parenchymal and non-parenchymal cells from adult human liver-morphological and biochemical characteristics. J Clin Chem Clin Biochem 14: $527-532$. 
Citation: Zhuang XM, Lu C (2016) The Past, Current, and Future Application of Cryopreserved Human Hepatocytes in Drug Development. Int J Clin Pharmacol Pharmacother 1: 120. doi: https://doi.org/10.15344/2016/2456-3501/120

Page 3 of 3

6. Fry JR (1982) The metabolism of drugs by isolated hepatocytes. Q Rev Drug Metab Drug Interact 4: 99-122.

7. Loretz LJ, Li AP, Flye MW, Wilson AG (1989) Optimization of cryopreservation procedures for rat and human hepatocytes. Xenobiotica 19: 489-498.

8. Li AP, Roque MA, Beck DJ, Kaminski DL (1992) Isolation and culturing of hepatocytes from human liver. J Tiss Culture Methods 14:139-146.

9. Ruegg CE, Silber PM, Mughal RA, Ismail J, Lu C, et al. (1997) Cytochrome-P450 induction and conjugated metabolism in primary human hepatocytes after cryopreservation. In Vitro Toxicol 10: 217-222.

10. Li AP, Lu C, Brent JA, Pham C, Fackett A, et al. (1999) Cryopreserved human hepatocytes: characterization of drug-metabolizing enzyme activities and applications in higher throughput screening assays for hepatotoxicity, metabolic stability, and drug-drug interaction potential. Chem Biol Interact 121: 17-35.

11. Huang SM, Strong JM, Zhang L, Reynolds KS, Nallani S, et al. (2008) New era in drug interaction evaluation: US Food and Drug Administration update on CYP enzymes, transporters, and the guidance process. J Clin Pharmacol 48: 662-670.

12. Lu C, Li P, Gallegos R, Uttamsingh V, Xia CQ, et al. (2006) Comparison of intrinsic clearance in liver microsomes and hepatocytes from rats and humans, Evaluation of free fraction and uptake in hepatocytes. Drug MetabDispos 34: 1600-1605.

13. Shibata $Y$, Takahashi H, Chiba M, Ishii $Y$ (2002) Prediction of hepatic clearance and availability by cryopreserved human hepatocytes: an application of serum incubation method. Drug Metab Dispos 30: 892-896.

14. Bachmann K, Byers J, Ghosh R (2003) Prediction of in vivo hepatic clearance from in vitro data using cryopreserved human hepatocytes. Xenobiotica 33: 475-483.

15. Blanchard N, Hewitt NJ, Silber P, Jones H, Coassolo P, et al. (2006) Prediction of hepatic clearance using cryopreserved human hepatocytes: a comparison of serum and serum-free incubations. J Pharm Pharmacol 58: 633-641.

16. Skaggs SM, Foti RS, Fisher MB (2006) A streamlined method to predict hepatic clearance using human liver microsomes in the presence of human plasma. J Pharmacol Toxicol Methods 53: 284-290.

17. Lu C, Miwa GT, Prakash SR, Gan LS, Balani SK (2007) A novel mode for the prediction of drug-drug interactions in humans based on in vitro cytochrome p450 phenotypic data. Drug Metab Dispos 35: 79-85.

18. Lu C, Berg C, Prakash SR, Lee FW, Balani SK (2008a) Prediction of pharmacokinetic drug-drug interactions using human hepatocyte suspension in plasma and Cytochrome P450 phenotypic data. II. In Vitroin Vivo Correlation with ketoconazole. Drug Metab Dispos 36: 1255-1260.

19. Lu C, Hatsis P, Berg C, Lee FW, Balani SK (2008b) Prediction of pharmacokinetic drug-drug interactions using human hepatocyte suspension in plasma and Cytochrome P450 phenotypic data. III. In Vitroin Vivo Correlation with fluconazole. Drug Metab Dispos 36: 1261-1266.

20. Mao JL, Mohutsky MA, Harrelson JP, Wrighton SA, Hall SD (2012) Predictions of Cytochrome P450-mediated drug-drug interactions using cryopreserved human hepatocytes: comparison of plasma and protein-free media incubation conditions. Drug Metab Dispos 40: 706-716.

21. Mao JL, Johnson TR, Shen ZZ and Yamazaki SJ (2013) Prediction of crizotinib-nidazolam interaction using the Simcyp population-based simulator: comparison of CYP3A time-dependent Inhibition between human liver microsomes versus hepatocytes. Drug Metab Dispos 41: 343-352.

22. Liu X, LeCluyse EL, Brouwer KR, Gan L-SL, Lemasters JJ, et al. (1999a) Biliary excretion in primary rat hepatocytes cultured in a collagen-sandwich configuration. Am J Physiol 277: G12-G21.

23. Liu X, LeCluyse EL, Brouwer KR, Lightfoot RM, Lee JI, et al. (1999) Use of $\mathrm{Ca} 2+$ modulation to evaluate biliary excretion in sandwich-cultured rat hepatocytes. J Pharmacol Exp Ther 289: 1592-1599.

24. Guo C, Yang K, Brouwer KR, St Claire RL, Brouwer KL (2016) Prediction of altered bile acid disposition due to inhibition of multiple transporters: An integrated approach using sandwich-cultured hepatocytes, mechanistic modeling, and simulation. J Pharmacol Exp Ther. 358: 324-333.

25. Yang K, Guo C, Woodhead JL, St Claire RL, Watkins PB, et al. (2016) Sandwich-Cultured Hepatocytes as a Tool to Study Drug Disposition and Drug-Induced Liver Injury. J Pharm Sci 105: 443-459.
26. Zhuang XM, Shen GL, Xiao WB, Tan Y, Lu C, et al. (2013) Assessment of the roles of P-glycoprotein and cytochrome P450 in triptolide-induced liver toxicity in sandwich-cultured rat hepatocyte model. Drug Metab Dispos 41: 2158-2165.

27. Schepers A, Li C, Chhabra A, Seney BT, Bhatia S (2016) Engineering a perfusable 3D human liver platform from iPS cells. Lab Chip 16: 2644-2653.

28. Nguyen DG, Funk J, Robbins JB, Crogan-Grundy C, Presnell SC, et al. (2016) Bioprinted 3D primary liver tissues allow assessmentof organ-level responseto clinical drug induced toxicity in vitro. PLoSONE 11: e0158674.

29. Bell CC, Hendriks DF, Moro SM, Ellis E, Walsh J, et al. (2016) Characterization of primary human hepatocyte spheroids as a model system for drug-induced liver injury, liver function and disease. Sci Rep 6: 25187.

30. Schaefer M, Schänzle G, Bischoff D, Süssmuth RD (2016) Upcyte Human Hepatocytes: a Potent In Vitro Tool for the Prediction of Hepatic Clearance of Metabolically Stable Compounds. Drug Metab Dispos 44: 435-444. 